\title{
CHANGES IN EMG AND FINGER FORCE WITH REPEATED HANGS FROM THE HANDS IN ROCK CLIMBERS
}

\author{
Phillip B. Watts $\ddagger$, Randall L. Jensen $\ddagger$, Sara M. Agena ${ }^{\dagger}$, John A. Majchrzak ${ }^{\dagger}$, Rebecca
}

A. Schellinger ${ }^{\dagger}$, Cory S. Wubbels ${ }^{\dagger}$

Exercise Science Laboratory, Department of Health, Physical Education and

Recreation, Northern Michigan University, Marquette, MI, USA

‡Denotes professional author, ${ }^{\dagger}$ denotes graduate student author

\begin{abstract}
Int J Exerc Sci 1(2): 62-70, 2008. The nature and degree of fatigue in muscles that control finger position during repeated sustained efforts in rock climbing have not been described. The purpose of this study was to identify changes in maximum hang time and forearm electromyogram (EMG) during repeated maximum duration hangs from a simulated rock feature. A second objective was to determine the effect of different recovery times between hangs upon changes in finger force. Five experienced rock climbers performed 2 test sessions on different days in a randomized order. Each session involved 8 repetitions of a maximum duration hang with either 1 min (R1) or 3 min (R3) resting recovery between hangs. Finger force (FF) was measured for the right hand via a piezoelectric force sensor fitted with a plate to accept the distal digits of four fingers. Peak EMG (EMGPK) and EMG Area (EMGAREA) were recorded from the anterior forearm for each hang and standardized as percent of maximum FF EMG prior to statistical analysis. Hang duration progressively decreased over repetitions but tended to plateau around repetition 5 for both R1 and R3 conditions. A significant difference was found for both recovery conditions and repetitions $(p<.05)$ as well as a significant interaction of the two factors $(p<.05)$ for hang duration. There were no significant differences for EMGPK among repetitions or between recovery conditions ( $p>.05)$. EMGAREA decreased initially with repeated hangs during both R1 and R3 but this trend tended to plateau at repetition 3 for the R3 condition. A significant difference was found in EMGAREA for both recovery conditions and trials $(p<.05)$ as well as a significant interaction of the two factors $(\mathrm{p}<.05)$. Mean FF decreased between pre- and post-hangs for both R1 and R3 however the difference was not significant. It was concluded that the overall decline in hang time is less with 3 min recovery vs 1 min recovery between hangs. Peak EMG does not appear to change despite this evidence of fatigue. A $3 \mathrm{~min}$ recovery interval between hang repetitions decreases the magnitude of fatigue experienced and enables a greater EMGAREA per hang.
\end{abstract}

KEY WORDS: Fatigue, hang-board, rock climbing

\section{INTRODUCTION}

Rock climbers often describe the cause of falling as a type of fatigue or inability to maintain contact force between the hands and the rock. Enoka and Stuart describe fatigue as "... a concept that encompasses a class of acute effects that impair motor performance" (3). In the context of their review, fatigue is defined as "... a general 
concept intended to denote an acute impairment of performance that includes both an increase in the perceived effort necessary to exert a desired force and an eventual inability to produce this force"(3). The nature of muscle fatigue that leads to a failure of contact in climbing remains unspecified.

It has been assumed that a loss of handgrip strength results in the decrease in contact force. In support of this, Watts et al. found a decrease in maximum handgrip strength following difficult climbing to the point of a fall in a group of 15 expert climbers (9). In this study, the post-climb maximum handgrip force averaged 22\% lower than pre-climb maximum force. Whether this degree of change in handgrip strength was directly related to the failure to continue the climbing task is unknown since the actual force required for maintaining contact was not measured.

Watts has subsequently suggested that contact force between the hand and the rock may be more associated with the effect of gravity on body mass than an actual concentric muscular force applied to the rock (12). Thus, a critical aspect of fatigue in climbing may not be a loss in handgrip force, but a loss in the ability to maintain a specific hand and finger configuration as gravity pulls the hand into the rock. This would be particularly significant during periods of sustained isometric effort as the climber works out the specific movements for further progress up the rock. Such sustained isometric effort is significant in climbing. Billat et al. have reported that static support represents $37 \pm 9 \%$ of climbing time during ascent of a difficult rock climbing route (1).
Watts suggests that measurement of strength via handgrip dynamometry may lack specificity with the hand positions and nature of muscle force in actual climbing (12). Contact with the rock only occasionally requires a pinch-type force between the thumb and fingers as in traditional handgrip dynamometry.

Further study into the specific nature and degree of fatigue in muscles that control finger force and hand position during sustained and repeated efforts in climbing is required to clarify these ideas. While the nature of muscle fatigue in climbing may not be well defined, it may be assumed that climbing-specific stress leads to adaptations that could improve resistance to fatigue.

Training routines employed by climbers are often of an interval nature with bouts of climbing-specific work followed by resting recovery periods (4). Interval training provides for a greater total amount of training work with less fatigue than continuous effort training. Various models exist for matching interval work time with recovery time for repetitions of sportspecific training tasks. A rest interval of three minutes is often recommended between repeated work bouts during resistance training (6). The effect of different recovery time between work bouts in climbing-specific training has not been studied.

Previous study has suggested that full recovery from exhaustive rock climbing is a prolonged process. Watts, et al. found mean handgrip force to remain significantly reduced from pre-climb level for up to 20 minutes following climbing to the point of falling (9). However, handgrip force did 
recover to a level significantly higher than the immediate post-climbing force within 10 minutes. This study did not observe subsequent climbing performance or muscle activation via electromyography, thus the degree to which partial recovery of handgrip force will effect further climbing and motor unit recruitment is not known. A recovery time of 10 minutes would be considered long for traditional interval training program designs or for the period between exercise sets in resistance training. The impact of shorter recovery times and various degrees of partial recovery on subsequent performance duration and muscle activation is not known.

A better understanding of climbing-specific fatigue and recovery from fatigue is necessary to further explore training program designs for improving climbing performance. Of interest would be the possibility that traditional interval training designs could be employed to specifically train the ability to sustain contact between the hands and the rock.

A variety of indoor devices are available to provide climbing-specific training. Many of these devices enable development of training workouts based upon historical designs employed in other activities, most specifically that of resistance training and interval training. A common and relatively inexpensive device is the hang-board (Figure 1 ). The basic hang-board design is a device that may be mounted above an open area to enable a climber to hang from it with body weight or added weight to increase the level of effort. Most commercially available hang-boards present a variety of hold shapes and sizes to simulate common rock features. Relatively sloping hold features are typically available to enable sustained hangs for maximum duration without risk of finger injury.

The purpose of this study was to identify changes in maximum hang time and forearm electromyogram during repeated hangs from a hang-board. A second objective was to determine the effect of short versus long recovery time on subsequent hang times and finger force.

\section{METHOD}

\section{Participants}

Five experienced rock climbers volunteered and signed informed consent to serve as subjects in the study. The mean $( \pm S D)$ age, height, and weight of the subjects were $22.4 \pm 2.2$ years, $166.4 \pm 8.4 \mathrm{~cm}$, and $62.1 \pm 11.2$ $\mathrm{kg}$ respectively. Each subject was provided an orientation and practice session to become familiar with the procedures of the study and the specific hang-board task.

\section{Protocol}

The study design required a subject to perform eight maximum duration hangs from the hands with full body weight. The hangs were performed on a hang-board training device that is employed by some rock climbers (Figure 1, Metolius Simulatorтm). The hand contact feature on the hang-board was a gradually sloping hold that accepted all three digits of the four fingers of both hands. The specific hand position would be described by rock climbers as a sloping open grip (Figure 2).

Two test sessions per subject were conducted on different days and at approximately the same time of day in a randomized order. Each session involved 
the subject performing 8 repetitions of a maximum duration hang with either 1 minute (R1) or 3 minutes (R3) of resting recovery between repetitions. The 8 repetition count was selected since earlier study in our laboratory had indicated a plateau in hang time after 4-6 repetitions. The 3 minute resting recovery duration was selected as comparable to the recommended recovery between exercises in traditional resistance interval training (6). The 1 minute resting recovery duration was selected to provide a significantly shorter recovery as a comparison to the 3 minute recovery. The duration of each hang repetition was recorded to the nearest 0.1 second via a digital stopwatch. A hang was defined as the time from the initial point of full body weight support from the hangboard until contact with the hand-board was broken by a fall.

Prior to and within 60 seconds of completion of each set of 8 hangs, maximum finger force $(\mathrm{FF})$ was measured for the right hand via a piezoelectric force sensor fitted with a plate to accept the distal digits of four fingers in an open grip position (Figure 3). Force was applied to the plate/sensor via a 3-second maximal finger flexion contraction. Previous study has found this instrument to be reliable in repeated measurements (10). Only the right hand was tested since the measurement device could record from only one hand at a time, thus a time delay effect would occur with recording force from the other hand. Since the specific hang-board feature employed provided symmetry during the hangs (Figure 2), it was assumed that the effect would be equivalent on each hand/arm.

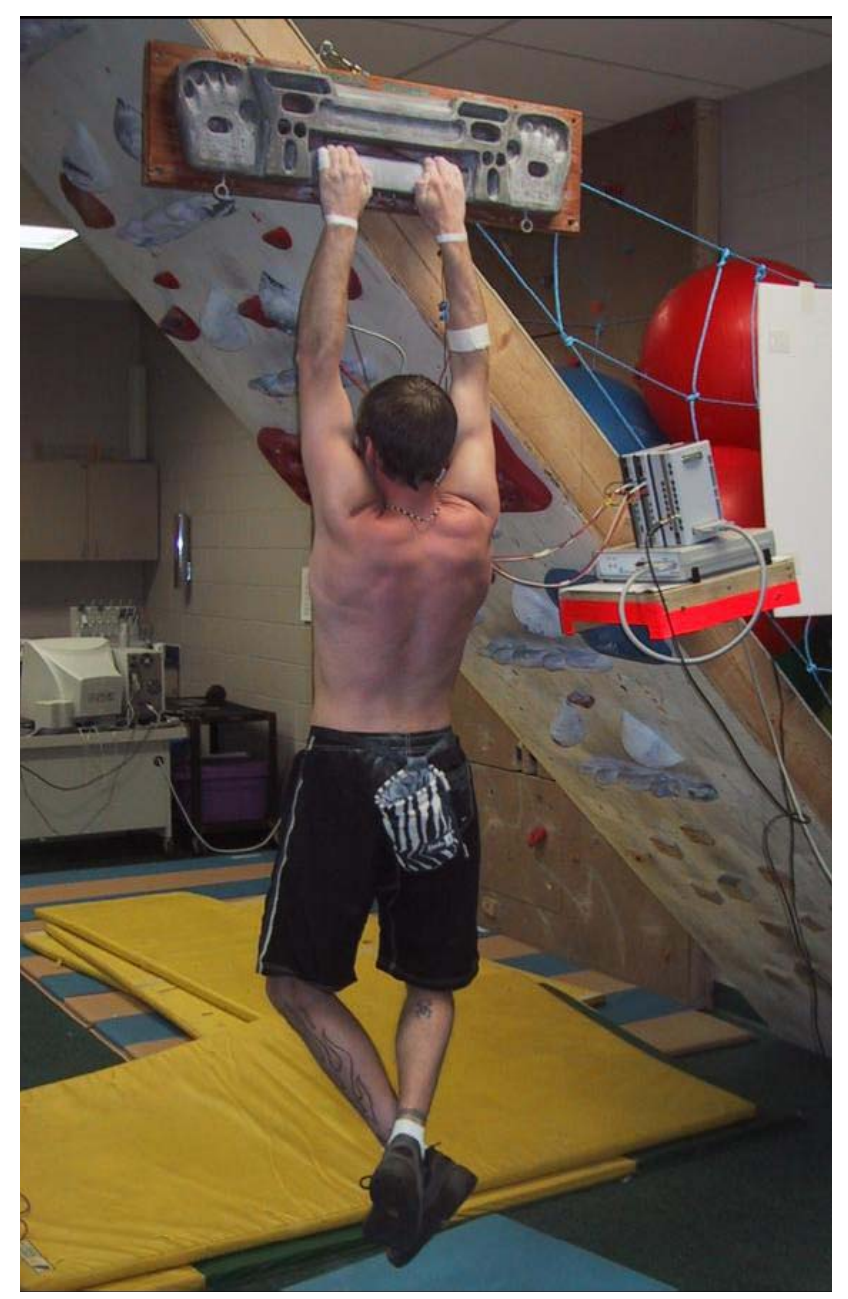

Figure 1. Subject position on the hang-board.

Electromyograms were recorded from the anterior forearm via surface electrodes (Blue Sensor; Medicotest A/S, Denmark). Previous study by Koukoubis, et al. has indicated immediate and sustained EMG activity in the anterior forearm during a climbing-type movement (5). One electrode was placed $1 / 3$ of the linear distance from the medial epicondyle of the humerus to the styloid process of the radius and a second electrode two $\mathrm{cm}$ distal along the same line according to Davies (2). A ground electrode was affixed at the olecranon process. Impedance between electrodes was

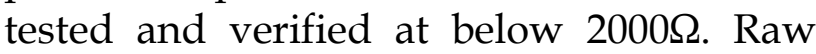
EMG data were recorded at $500 \mathrm{~Hz}$ (Biopac 
MP100). The raw EMG signals were integrated via root mean squared (RMS) over 50 samples and peak values subsequently determined via Acqknowledge version 3.5.6 software (Biopac Systems, Inc.). EMG was acquired continuously during each hang trial and Peak amplitude (ЕМGрк) and area (EMGaRea) determined for each hang repetition. EMGPK was standardized as a percent of maximum FF EMG prior to statistical analysis. EMG and force data were acquired simultaneously during the FF tests.

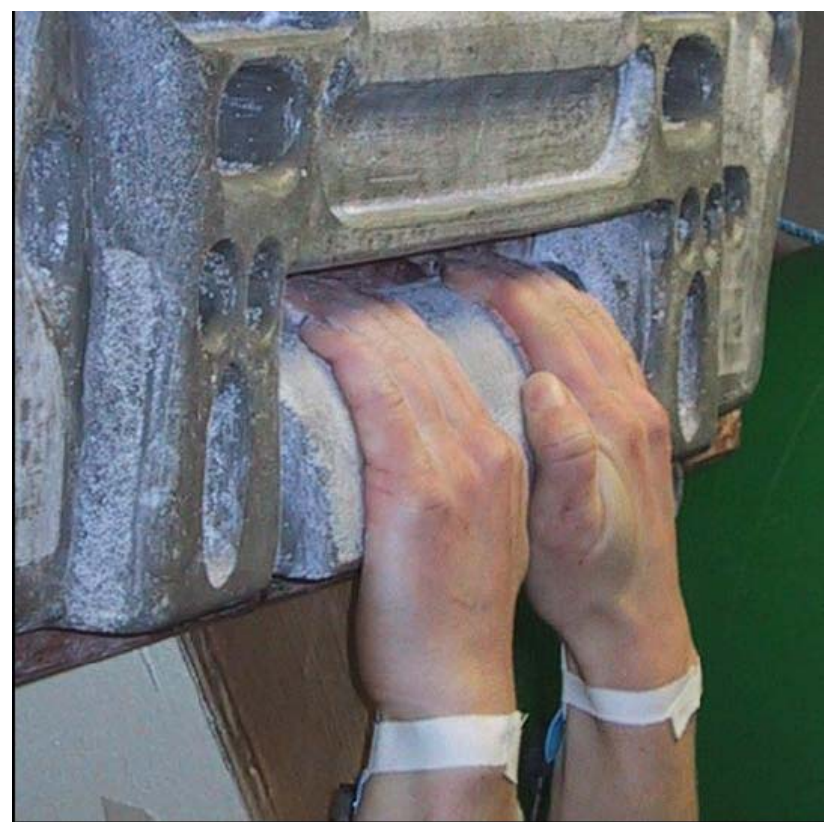

Figure 2. Detail of hand positions on the sloping open grip hold of the hang-board.

\section{Statistical Analysis}

Comparisons among repetitions and between recovery conditions were made via two-way repeated measures ANOVA (SPSS).

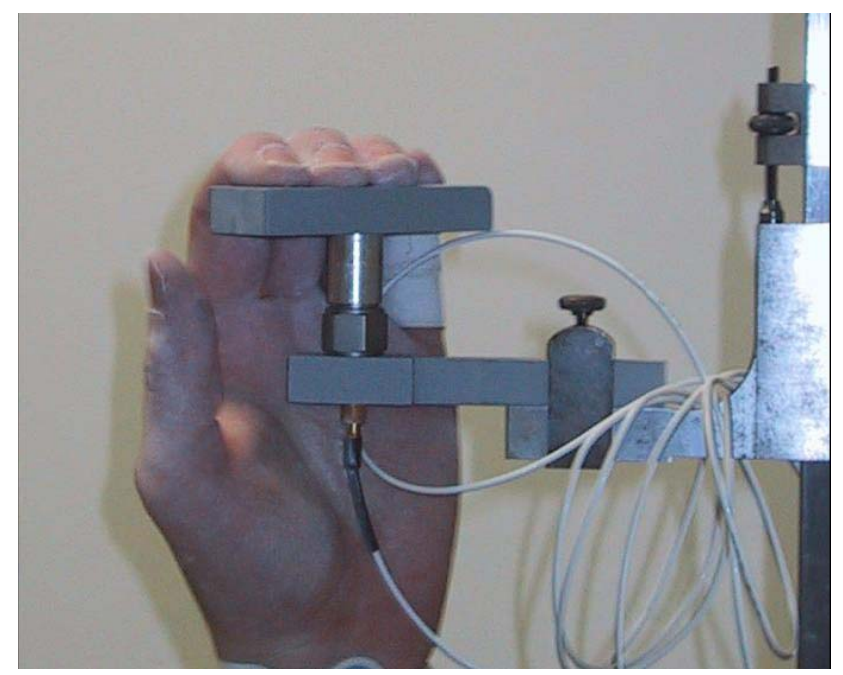

Figure 3. Detail of apparatus and hand position for measurement of finger force.

\section{RESULTS}

Subject weight did not change significantly over the course of the study $(62.0 \pm 11.2$ vs $61.6 \pm 11.2 \mathrm{~kg}$ for R1 vs R3). Mean hang duration initially decreased over repetitions and tended to plateau for both R1 and R3 conditions (Figure 4). Mean hang durations (SD) for repetitions 1 vs 8 were $40.7 \pm 15.2$ vs $11.0 \pm 3.9 \mathrm{sec}$ for R1 and $36.3 \pm 12.8$ vs $24.7 \pm 10.7 \mathrm{sec}$ for R3 respectively. Two-way repeated measures ANOVA indicated a significant difference for both recovery conditions and repetitions $(\mathrm{p}<.05)$ as well as a significant interaction of the two factors $(p<.05)$ for hang duration.

In spite of the decrease in hang time across hangs there were no significant differences for EMGPK among repetitions or between R1 and R3 (P>.05; Fig. 5). 


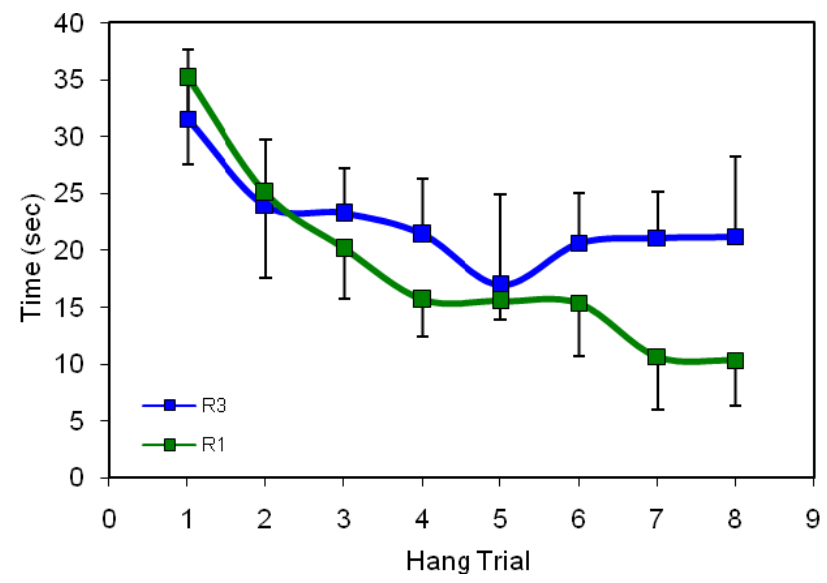

Figure 4. Mean $( \pm S D)$ hang duration (Time) for 8 repetitions with either 3-min (R3) or 1-min (R1) rest recovery between hangs.

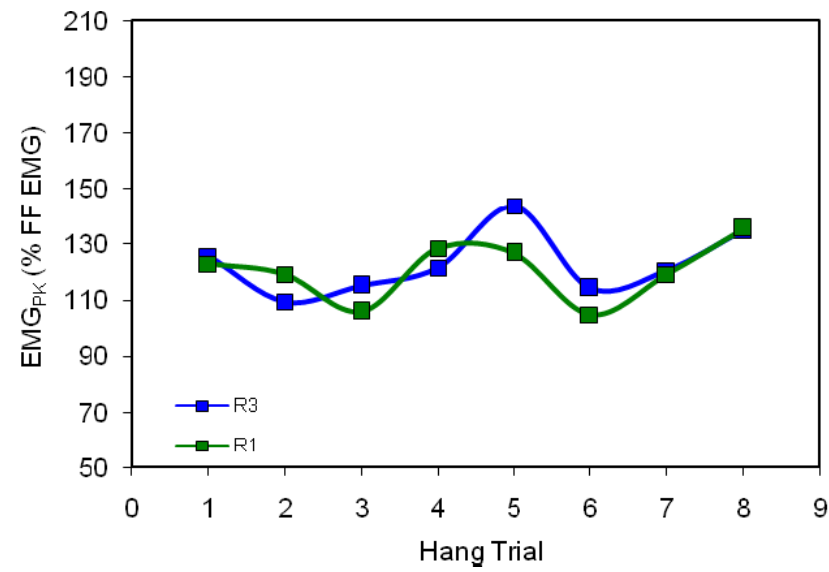

Figure 5. Mean EMG ${ }_{\mathrm{PK}}$ amplitude for 8 repetitions with either 3-min (R3) or 1-min (R1) rest recovery between hangs as a percentage of the pre-hang.

EMGAREA decreased initially for both R1 and R3 across repetitions and tended to plateau after repetition 3 for the R3 condition (Fig. 6). Two-way repeated measures ANOVA indicated a significant difference for EMGAREA between R1 and R3 across repetitions $(p<.05)$ as well as a significant interaction of the two factors $(p<.05)$. Total hang time $(147.7 \pm 19.2$ vs $179.8 \pm 31.1 \mathrm{sec})$ and total EMGAREA $(27.8 \pm 6.6$ vs $37.5 \pm 9.8$ units) differed significantly between R1 vs R3 respectively $(p<.05)$.
Finger force decreased between pre- and post-hangs for both R1 $(21.5 \pm 12.5 \mathrm{~kg}$ vs $16.3 \pm 10.8 \mathrm{~kg})$ and $\mathrm{R} 3(23.3 \pm 9.3 \mathrm{~kg}$ vs $20.5 \pm 7.8 \mathrm{~kg}$ ) however this change was not significant (see Figure 7).

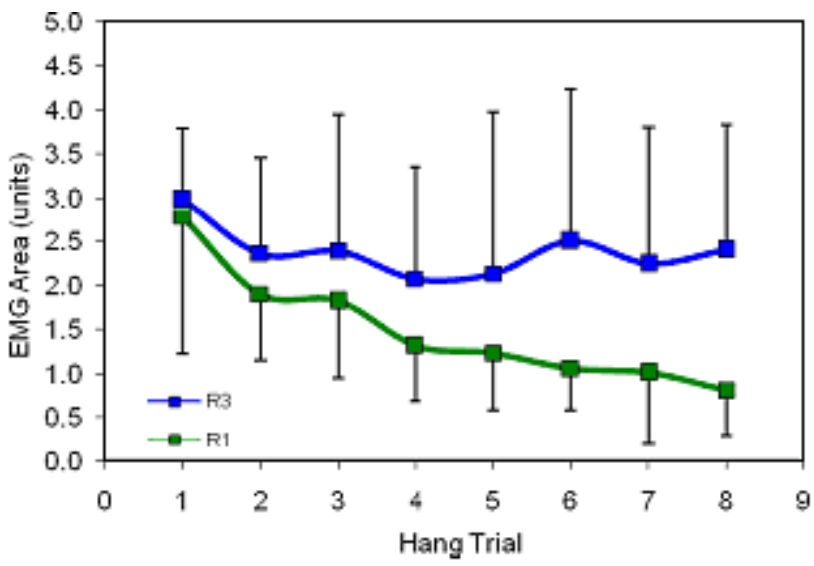

Figure 6. Mean $\mathrm{EMG}_{\mathrm{AREA}}$ for 8 repetitions with either 3-min (R3) or 1-min (R1) rest recovery between hangs.

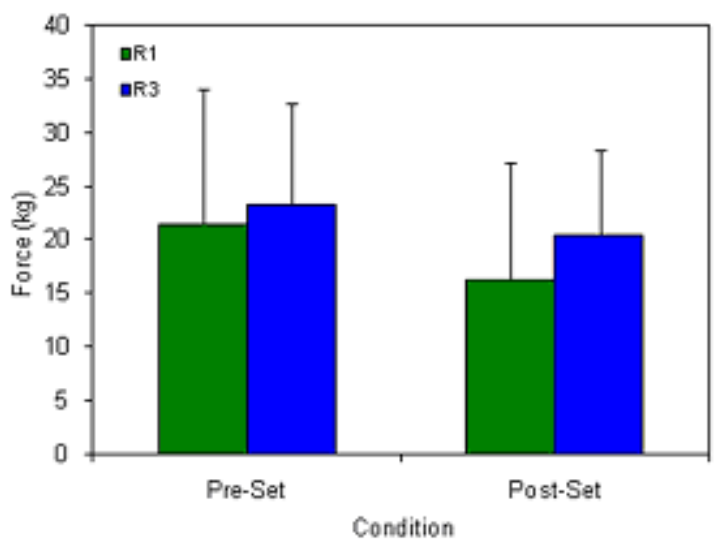

Fig. 7. Mean maximum finger curl force before (PreSet) and after (Post-Set) 8 hang repetitions with either 3-min (R3) or 1-min (R1) rest recovery.

\section{DISCUSSION}

This study was conducted to observe changes in maximum hang time and forearm electromyogram during repeated 
hangs from a simulated rock feature. A second objective was to determine the effect of short versus long recovery time on subsequent hang times and finger force.

The results indicate that, although hang time decreases with repeated hangs, there is a tendency for hang time to plateau across repetitions. This is particularly true when the rest interval between repetitions is three minutes. The plateau begins to occur at repetition 4 (figure 4) and is well established by repetition 6 where the mean hang time was $33.3 \pm 16.3 \%$ of repetition 1 . It should be noted that, since recovery time was maintained as hang time decreased, the ratio of recovery time to hang time increased across repetitions until the plateau occurred. For the 3-minute recovery condition, it appears that, once hang time decreased to a point approximating $33 \%$ of maximum hang time, the rest period provided adequate recovery to repeat the task at this level.

Conversely, for the 1-minute recovery trial, attainment of a plateau is less clear and the $33 \%$ of maximum hang time level is not sustainable. The result is that less total muscle work is performed when a 1-minute recovery is employed versus the 3-minute recovery. This is also reflected in the significantly different EMGAREA values (figure 6) and the total hang times between recovery conditions.

Our subject sample was too small to test for a climbing-ability effect on hang time and resistance to fatigue. Quaine, et al. have shown that experienced climbers significantly resist fatigue better than untrained subjects during repetitions of a finger force task at $70-80 \%$ of maximum effort (7). It would be interesting to see if high-ability climbers experience less decrease in hang time as the plateau is attained.

It is interesting that the EMGPK did not change across repetitions for either recovery time condition (figure 5). It could be that once a given level of recruitment has been attained, the muscle systems are unable to counter the effects of fatigue. It is possible that the observed EMGpK values represent a rise to maximum voluntary motor unit activation over the course of each hang as some motor units fatigue. Thus, once the maximum level of activation is reached, the level of tension development in the muscles may become inadequate to maintain hand/finger position against the constant resistance force produced by the hanging subject and the climber falls. Residual fatigue in some motor units between hangs could result in the decrease in time for progression to the point of maximal recruitment as reflected in the decrease in hang time across the initial hangs.

Although less total work time and less EMGAREA occurs with a 1-minute recovery period between repetitions, the resultant level of fatigue following completion of 8 repetitions is greater than with a 3-minutes recovery. This information has implications relative to training program design. If the goal of a specific training lesson is to generate a high level of fatigue, then a 1minute recovery interval would be indicated. If the goal is to perform a high volume of work, then a 3-minute recovery interval would be best. The climbingspecific adaptations to these different stresses have not been studied. 
The observation that hanging from the hang-board with body mass produced a higher EMGPK than that recorded during a maximum-effort isometric finger flexion is troublesome (figure 5). This phenomena indicates that a greater muscle mass is recruited during the sustained hangs. We have also observed this when comparing climbing EMG with maximum handgrip dynamometry EMG (13). It has been demonstrated that EMG amplitudes recorded at the endurance limit are less than EMG amplitudes recorded during maximum voluntary contractions (14). It is possible that the device employed to measure finger force (figure 3) represents a submaximal effort, or that the surface electrode configuration used in our study records from additional muscle not activated during the finger force test. This merits further study.

We did not increase the load by adding weight above the subject's body weight. Weighted hangs may be more similar to resistance training models that use high resistance and low repetitions. Earlier unpublished observations in our lab indicate that hang-times are reduced when additional weight above body weight is supported by the subject during a hang. Although first repetition hang-time is shorter, hang-time still plateaus with repeated trials even when an additional 22 $\mathrm{kg}$ of weight is supported by the subject during a hang. The finding of consistent EMGPK amplitude at the point of falling from the hold was also observed with weighted hangs (8).

Maximum hang time decreases with repeated hangs, but tends to level off after 3-5 repetitions with three minutes of recovery between hangs. The decline in hang time with repeated hangs is less with a three minute recovery versus a one minute recovery. A set of eight maximum duration hangs results in a decrease in maximum finger force, but this decrease is less with a three minute recovery between repetitions. Peak EMG amplitude does not appear to change across repetitions for either recovery condition despite evidence of fatigue in hang time and finger force. The three minute recovery between repetitions enables a higher total work time and greater EMG area during a set of eight maximum duration hangs. Whether this increase in muscle activation and work time enhances any training adaptation is unknown.

\section{REFERENCES}

1. Billat V, Palleja P, Charlaix $T$, et al. Energy specificity of rock climbing and aerobic capacity in competitive sport rock climbers. J Sports Med Phys Fit 35: 20-24, 1995.

2. Davies JF. Manual of surface electromyography. WDAC Technical Report \#59-184, 1959; in Selective Topics in Surface Electromyography for Use in the Occupational Setting: Expert Perspectives. U.S. Department of Health and Human Services, 1992.

3. Enoka RM, Stuart DG. Neurobiology of fatigue. J Appl Physiol 72(5): 1631-1648, 1992.

4. Horst EJ. Training for Climbing. Falcon - Globe Pequot Press, 2003.

5. Koukoubis TD, Cooper LW, Glisson RR, Seaber AV, Feagin JA. An electromyographic study of arm muscles during climbing. Knee Surg Sports Traumatol Arthrosc 3: 121-124, 1995.

6. McArdle WD, Katch FI, Katch VL. Exercise Physiology, 6th Edition. Lippincott, Williams and Wilkins, 2007. 
7. Quaine F, Vigouroux L, Martin L. Finger flexors fatigue in trained rock climbers and untrained sedentary subjects. Int J Sports Med 24: 424-427, 2003.

8. Watts PB, Martin DT, Baumgarten D. Factors related to performance on the Metolius Simulator. Proceedings of The 1989 International Olympic Committee World Congress on Sport Sciences, Colorado Springs, Colorado, USA, 1989.

9. Watts PB, Newbury V, Sulentic J. Acute changes in handgrip strength, endurance, and blood lactate with sustained sport rock climbing. J Sports Med Phys Fitness 36: 255-260, 1996.

10. Watts PB, Daggett M, Gallagher P, Wilkins B. Metabolic responses during sport rock climbing and the effects of active versus passive recovery. Int J Sports Med 21: 185-190, 2000.

11. Watts PB, Jensen RL. Reliability of peak forces during a finger curl motion common in rock climbing. Meas Physical Ed Exer Sci 4: 263-267, 2003.

12. Watts PB. Physiology of difficult rock climbing: a review. Eur J Appl Physiol 91: 361-372, 2004.

13. Watts PB, Jensen RL, Gannon E, Kobeinia R, Maynard J, Sansom J. Forearm EMG during rock climbing differs from EMG during handgrip dynamometry. Int J Exerc Sci 1(1): 4-13, 2008.

14. West W, Hicks A, Clements L, Dowling J. The relationship between voluntary electromyogram, endurance time and intensity of effort in isometric handgrip exercise. Eur J Appl Physiol 71: 301-305, 1995. 